\title{
The use of the power law for designing wireless networks
}

\author{
Andrzej Paszkiewicz ${ }^{1, *}$ \\ ${ }^{1}$ Department of Complex Systems, The Faculty of Electrical and Computer Engineering, Rzeszow \\ University of Technology, al. Powstańców Warszawy 12, 35-959 Rzeszów, Poland
}

\begin{abstract}
The paper concerns the use of the scale-free networks theory and the power law in designing wireless networks. An approach based on generating random networks as well as on the classic Barabási-Albert algorithm were presented. The paper presents a new approach taking the limited resources for wireless networks into account, such as available bandwidth. In addition, thanks to the introduction of opportunities for dynamic node removal it was possible to realign processes occurring in wireless networks. After introduction of these modifications, the obtained results were analyzed in terms of a power law and the degree distribution of each node.
\end{abstract}

\section{Introduction}

The last half a century is a period of an intensive development of different types of networks. Initially it was communication and energy infrastructure, but in the next step phone networks appeared as well, and currently computer networks exist. We should also remember about social networks. The importance of this type of structure has increased along with the development of the Internet network. The modeling and methods of supporting of the design process are therefore an important part of the work area of engineers and scientists. The paper is primarily focused on designing wireless networks and using a power law for this purpose.

Computer networks like other natural and artificial systems should be treated like complex systems [1-3]. This group includes both biological organisms, social relations and infrastructure including computer networks. For many years such systems have been treated as simple systems and specific methods and means designed for these systems have been applied to them. However, the properties of complex systems require the utilization of suitable mechanisms and models based on fractal structures, power law, non-extensive statistics, etc. $[4,5]$. Of course, we must be aware that due to the complexity of the complex systems design problem, their individual properties in the proposed solutions, algorithms and models should be gradually taken into account [6-8].

Unfortunately, for many years, the complex networks have been treated as regular or random structures. In case of regular structures, it was assumed that the degree of each node is the same. Thanks to these properties, the regular networks are characterized by a high clustering coefficient, but have a long average distance between nodes. Such an approach

\footnotetext{
Corresponding author: andrzejp@prz.edu.pl
} 
could make sense only in rarely met special cases. However, such an assumption made it easier to formulate statements and achieves the appropriate topological parameters. This approach recommended and even enforced using specific structures e.g. rings, grids, binary trees, etc. for computer networks and their many variations and modifications. The other, different way to create merging structures was the adoption of the random model. This was the result of Paul Erdos and Alfred Renyi's statement in 1959 that such systems can be modeled by random connecting nodes between each other and they have a short average distance between the vertices [9]. As a consequence of this, such a model was generally used, and moreover it turned out that the systems based on this model were characterized by the Poisson distribution in relation to the degree of each vertex (network node) $[10,11]$. That system had the symptoms of a democratic one in which majority of nodes have a similar number of connections. As a result, despite the randomness, it wasn't possible to have structures with specific centers or nodes having much more connections than other vertices. This resulted from the assumption that together with the increase of edges attached to the vertex, the probability of joining other ones is decreasing. One of the variants of the random model are mechanisms based on genetic algorithms, in which a set of selected individuals from the population is put to the pseudo random operations of crossover and mutation [12]. During the study of functioning of unnatural systems and the behavior of many natural systems, it was found that their connection, communication structure and social relations as well, they have the characteristics described by the power law [13-15]. The use of this phenomenon in the design of the network infrastructure was initiated by the preferential attachment algorithm presented by Albert-László Barabási and Albert Réka [16]. This algorithm applies to so-called scale-free network, in which the increase of the node's value depends on its current value - this is exactly described in the following part.

This paper presents the results of simulations connected with the introduction of improvements in the form of a modification of the classical preferential attachment algorithm. The purposes of these actions were to make the process of modeling of computer networks, especially wireless networks, more realistic.

\section{Power Law and scale-free networks}

The power law has accompanied us from the beginning of the time. However, relatively recently it has been used to describe phenomena occurring in artificial systems such as e.g. computer networks. For the first time, the power law was observed in the science by B.G. Buelnger in 1729 during research on stress relaxation in the materials. A significant contribution to the research in the field of power law was made by the Italian sociologist and economist Vilfredo Pareto, who studied, among others, distribution of income and size/level of salaries in society. According to the power law, the degree distribution of vertices is a strongly decreasing function with the so-called heavy tail. However, in the double logarithmic scale, they form straight lines (Fig. 1).

Thus, in the contrast to the democratic distribution of connections known from the random networks, the power law describes systems in which several centers of the so-called hubs are dominators. This type of networks are called scale-free networks [17]. In these networks the probability of connection between the new node and each node belonging to the network depends very much on the number of edges possessed by these nodes and for the $k$-th vertex is expressed by:

$$
P(k) \sim k^{-\gamma}
$$


a)

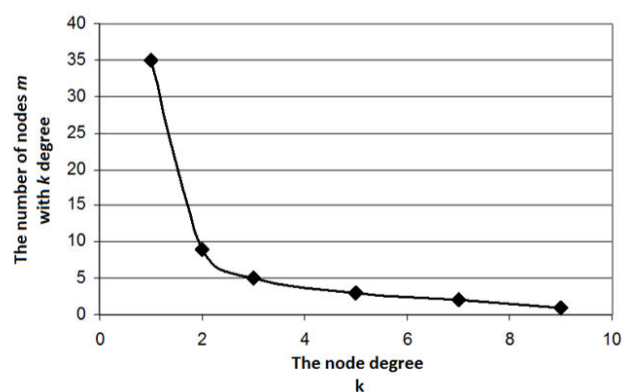

b)

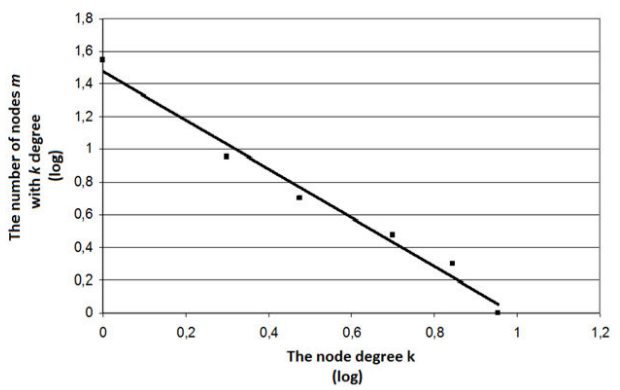

Fig. 1. The probability distribution of connecting to the node: a) the distribution corresponding to the power law; b) the distribution corresponding to the power law in a double logarithmic scale.

The use of relations coming from a power law in computer science was initiated in 1998 in Hawoong Jeong and Réka Albert's works from the University of Notre Dame [18]. These scientists began project of website network mapping. Initially, they assumed that results they obtained would confirm dependences that are characteristic for a random network. However, researches has shown that a small number of pages have multiple connections. Whereas, more than $80 \%$ of pages had four connections. From this perspective, it turned out that the system of links between Web pages perfectly fits into relations compatible with a power law. The result of this discovery was showing scale-free feature other networks, which similarly show the power distribution of vertex degrees. This relationship can be very easily observed in a social network, in which a person with a large number of friends is attracting new ones faster $[19,20]$. The same is observed in the structure of e-mail connections [14] between different domains, and in the structure of the Internet as well. There are nodes (hubs) in this network that have a particularly large number of connections with others, and also represent the intermediate point for a large number of flows between autonomous systems [13]. These are, of course, selected examples from the area of computer science, where power law and scale-fee networks are visible. Similar examples can be found in other areas of life and also in different fields of science. The income of states, the income of citizens, the distribution of earthquakes as well as the frequency of words in the text, etc. are shaped according to the power law. Taking it into account, relations resulted from a power law should form the basis of currently created models and algorithms of designing connection structures.

An important advantage of systems built in accordance with the power law is their resistance to accidental failures. Due to the fact that many nodes have a small number of connections with other nodes, their accidental damage will not significantly affect the functioning of the entire structure. On the other hand, what is the advantage may be some kind of threat, so there are particularly important centers, which damage will cause the degradation of the entire system. Therefore, they should be especially well protected. However, the problem of protection of these nodes is not the subject of this work.

\section{Random structures vs. the preferential attachment algorithm}

Wireless networks are characterized by very large dynamics of changes in the structure. At first glance it would seem that the appearance of new nodes (users) in the wireless network has a random character. However, after a deeper analysis, it turns out that also in wireless networks there are specific centers (hubs), which link a much larger number of users than others. Therefore, it appears reasonable to use models based on a power law during designing a wireless network. 
At the beginning, experiments were carried out, in which the degree distribution of vertices for the random mechanism and based on preferential attachment (the power law) was compared. For the first case, the obtained results are shown in Fig. 2.

a)

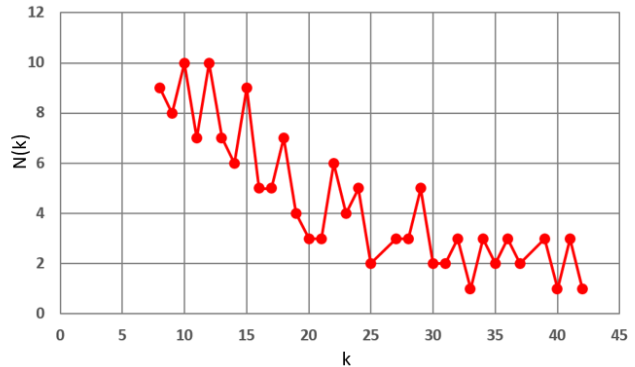

b)

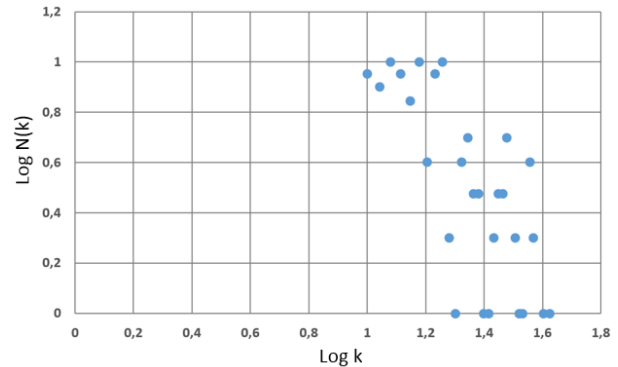

Fig. 2. The probability distribution of connecting to the node (for the random mechanism): a) the distribution corresponding to the power law; b) the distribution corresponding to the power law in a double logarithmic scale.

The process of preferential attachment consists in binding nodes based on the rule that the higher probability, the greater the chance that more will be attached to a given node. This phenomenon is characteristic for scale-free networks. This mechanism was proposed by Barabási and Albert [21]. On its basis, a program generating a graph was created, which presents a scale-free network. The classic algorithm of modeling scale-free network is presented below:

- Initially, we create the relatively small number of nodes (corresponding APs).

- We introduce another new node (representing a client). Probability that the new node (client) is connected with the $i$-th node (access point) depends on the degree of node $\left(k_{\mathrm{i}}\right): \quad P\left(k_{i}\right)=k_{i} / \sum_{j} k_{j}$.

- We continue to add new nodes (clients), until requested quantities.

Examples of wireless network designing results obtained by the action of the classic preferential attachment algorithm were illustrated on Fig. 3.

a)

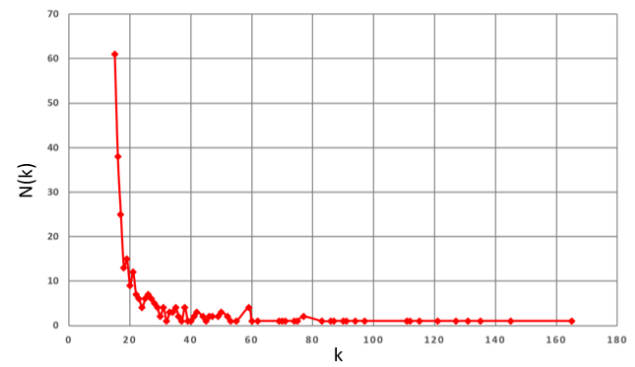

b)

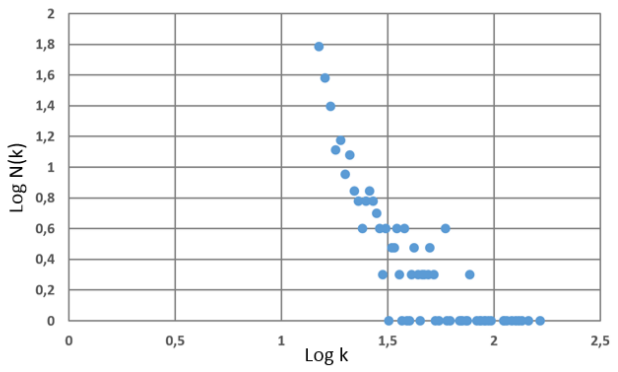

Fig. 3. The probability distribution of connecting to the node (for the classic preferential attachment algorithm): a) the distribution corresponding to the power law; b) the distribution corresponding to the power law in a double logarithmic scale.

As shown in the charts above, the values of individual nodes degrees are close to each other in the random model. Thus, the standard deviation is small in this case. On the other hand, in the case of a model based on preferential attachment the obtained results fit perfectly into relations resulting from the power law.

However, the adoption of the classical Barabási-Albert algorithm for creating scale-free networks does not take many important factors into account. Therefore, this model does not correspond to the real limitation of wireless networks. 


\section{The proposed solution}

In this paper a new approach to the designing of wireless networks based on the power law is proposed. This approach takes additional factors into account, such as limiting the bandwidth and dynamic removal of added nodes. Thanks to this, the modeled system is close to real conditions. The classic model based on the Barabási-Albert algorithm belongs to a group of incapacitated models [22]. These models assume that the hubs (concentrators) have no capacity limitations. In the case of the proposed solution, the limitation for both the available capacity for each node and the capacity (weight) of end users was introduced. Therefore, this approach is consistent with capacitated models [22]. This mechanism is based on an assumption that is compatible to the expression:

$$
C_{i}^{\max } \leq \sum_{j} c_{j}
$$

where $C_{i}^{\max }$ indicates the maximum capacity (weight) of the given node and $c_{j}$ is a value of traffic (weight) generated by the user (node). Thus, exceeding the sum of the edge weights of the added vertex relative to the specified value stops the adding of further edges. This situation corresponds to the maximum possibilities of transferring data through the node, that is, its capacity. Fig. 4 shows examples of wireless network design results obtained on the basis of operating of the improved preferential attachment algorithm that takes the capacitated model into account. The results confirm that the introduction of the capacitated model for designing wireless networks based on the preferential attachment algorithm has not changed the nature of the network. Still it has the character of scale-free network consistent with the power law.

a)

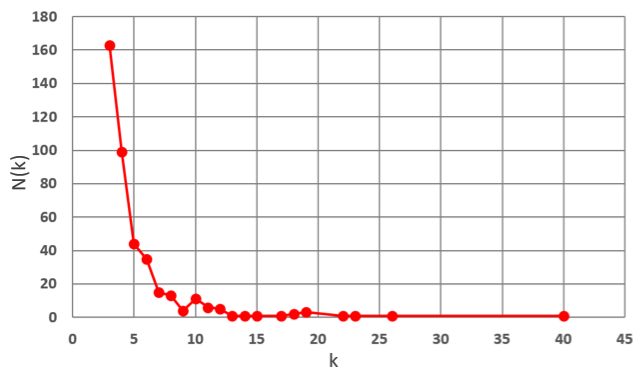

b)

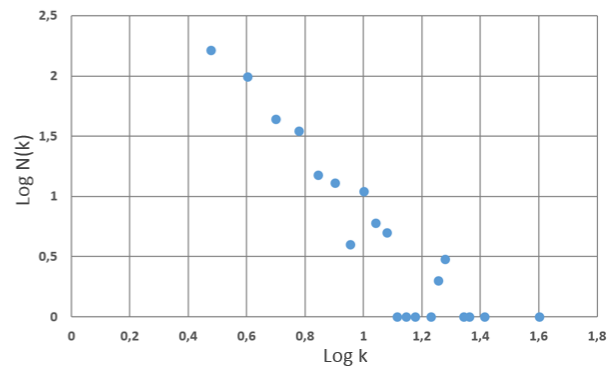

Fig. 4. The probability distribution of connecting to the node (for the capacitated model): a) the distribution corresponding to the power law; b) the distribution corresponding to the power law in a double logarithmic scale.

Another improvement in the proposed solution is consideration of both the appearance of dynamics nodes and their disappearance. Wireless networks are characterized by high dynamics of changes as to the number of current users and devices available (active) at the moment. The classical algorithm for creating scale-free networks does not take this factor into account. The aim of the simulations was to observe whether this change will influence the character of the modeled network. Despite the introduction of randomness of removal nodes, the network still retained its character compatible with the power law as shown in Fig. 5. 
a)

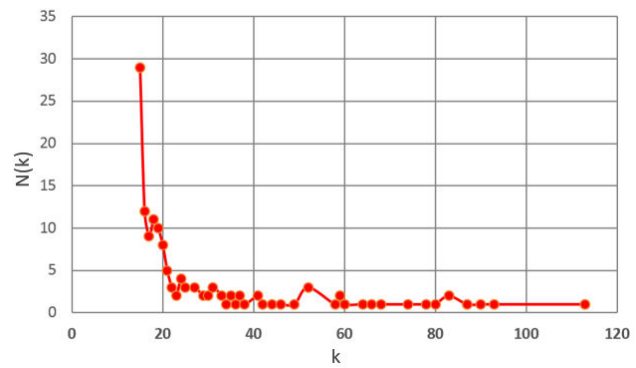

b)

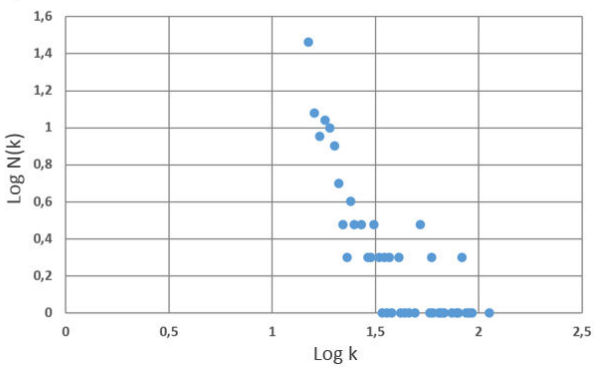

Fig. 5. The probability distribution of connecting to the node (by randomness of removal nodes): a) the distribution corresponding to the power law; b) the distribution corresponding to the power law in a double logarithmic scale.

So far, local wireless networks have usually been a form of a gateway that allows end users to communicate with the remaining network infrastructure (mostly wired) or to provide access to the Internet. However, more and more often wireless networks are an extensive network structure enabling communication between its elements. Individual wireless users (or stations) can provide various services to each other. Very often such services require stable transmission parameters over the entire data path. In this case, one should strive to ensure the best conditions for communication, especially with a large number of active devices/users. One of the possible mechanism to be used in this case is load balancing. Based on an algorithm of preferential attachment some modifications were made. The amount of flows between any pair of users passing through individual access nodes were analyzed. Thus, in this case a higher probability to establish new connections had those nodes through which the least number of paths passes between any pair of vertices. Of course, keeping all other assumptions resulting from the classical preferential attachment algorithm. The described modification is based on the expression:

$$
\min \sum_{(i, j) \in V} p_{i j}(x)
$$

where $p_{i j}$ represents the existence of the data flow path between any pair $i$ and $j$ in a given node. $p_{i j}$ can be set as 1 if there is a flow, and 0 if it is not. This assumption takes into account the mechanism of load balancing, and such an approach may have a positive effect in the case of networks with medium or high density of wireless nodes.

a)

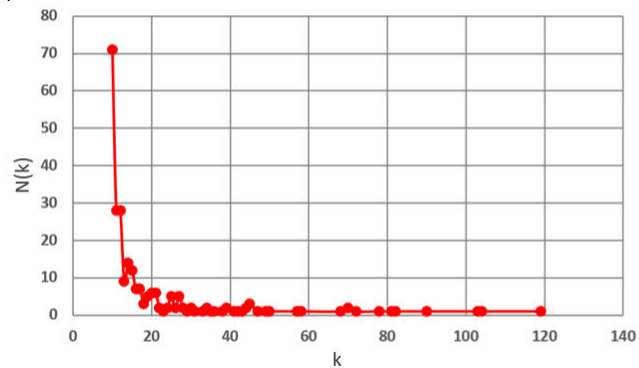

b)

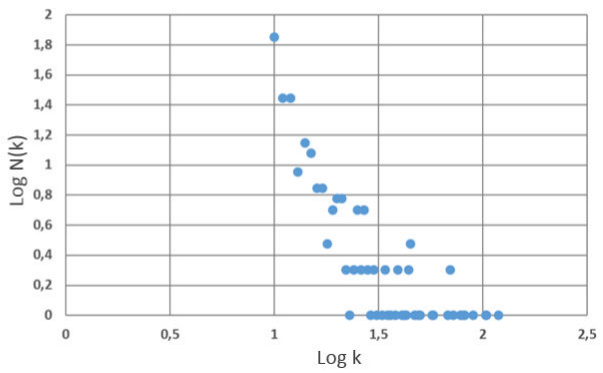

Fig. 6. The probability distribution of connecting to the node (by the mechanism of load balancing): a) the distribution corresponding to the power law; b) the distribution corresponding to the power law in a double logarithmic scale.

Moreover, Fig. 6 presents the results of a sample simulation of the modified algorithm. The results confirm that the introduction of modification taking into account the flow degree 
of the node to designing of wireless networks based on the preferential attachment algorithm has not changed the nature of the network.

\section{Conclusions}

The development of mobile technologies causes that it is necessary to search for effective models and mechanisms to support the design and analysis of the functioning of wireless networks. Wireless networks are complex systems. Therefore, appropriate models should be used for these systems, and they are taking into account the actual phenomena occurring in these systems. One of the mechanism that describes these phenomena is the power law. This law can be observed in many naturally occurring effects such as earthquakes, rainfall, topography as well as many human relationships, such as professional links, quoting articles, or wealth distribution in society. It was also noticed in many aspects of the operation of computer systems including software systems and computer networks. Therefore, it should be used during designing of wireless networks. As shown in this paper, the values of individual nodes degrees are close to each other in the random model. Thus, the standard deviation is small in this case. On the other hand, in the case of a model based on preferential attachment, the obtained results fit perfectly to relations resulting from the power law. However, the classical preferential attachment algorithm based on the power law, does not take into account many factors that affect the functioning of the real wireless networks. Thus, the bandwidth of limitations and the number of active users, and the load balancing mechanism were included into the algorithm. Therefore, the solutions proposed are some kind of an approximation of applied models to real conditions. Additionally, as a result of the simulations, it was shown that the changes do not modify the nature of the scale-free network. In future works, queuing mechanisms can be included, and the limitations of the power of mobile devices in developed models and algorithms.

\section{References}

1. J. Ladyman, J. Lambert, K. Wiesner, What is a complex system? European Journal for Philosophy of Science, 3(1), 33-67 (2013)

2. F. Grabowski, Nonextensive model of self-organizing systems. Complexity, Wiley, 18(5), 28-36 (2013)

3. G.M. Dimirovski (Ed.), Complex Systems. Relationships between Control, Communications and Computing. Studies in Systems, Decision and Control, 55 (2016)

4. G. Nicolis, C. Nicolis, Foundations of Complex Systems: Emergence, Information and Predicition (2012)

5. F. Grabowski, A. Paszkiewicz, M. Bolanowski, Wireless networks environment and complex networks, Analysis and Simulation of Electrical and Computer Systems, 324, 261-270 (2015)

6. Z. Gomółka, B. Twaróg, J. Bartman, B. Kwiatkowski, Improvement of Image Processing by Using Homogeneous Neural Networks with Fractional Derivatives Theorem, AIMS, Discrete and Continuous Dynamical Systems, Journal of American Institute of Mathematical Sciences, 505-514 (2011)

7. T. Pełech-Pilichowski, A hybrid algorithm for detecting changes in diagnostic signals received from technical devices, Proceedings of the Federated Conference on Computer Science and Information Systems, 321-327 (2011)

8. T. Pełech-Pilichowski, J.T. Duda, A two-level detector of short-term unique changes in time series based on a similarity method, Expert Systems 2015, 32(4), 555-561 (2015)

9. P. Erdős, A. Rényi, On Random Graphs I, Publicationes Mathematicae, 290-297 (1959) 
10. M.E.J. Newman, Random graphs as models of networks, Santa Fe Institute (2002)

11. M.E.J. Newman, S. Strogatz, D. Watts, Random graph models of social networks, Proceedings of the National Academy of Sciences of the United States of America, 99, 2566-2572 (2002)

12. S.N. Sivanandam, S.N. Deepa, Introduction to Genetic Algorithms, Springer-Verlag Berlin Heidelberg (2008)

13. G. Siganos, M. Faloutsos, P. Faloutsos, Ch. Faloutsos, Power laws and the AS-level internet topology, IEEE/ACM Transactions on Networking, 11(4), 514-524 (2003)

14. H. Ebel, L. Mielsch, S. Bornholdt, Scale-free topology of e-mail networks, Physical review E, 66(3) (2002)

15. S. Redner, Citation Statistics From More Than a Century of Physical Review, Physics Today, 58, 49-54 (2005)

16. R. Albert, A.L. Barabási, Statistical mechanics of complex networks, Review of Modern Physics, 74, 47-91 (2002)

17. A.L. Barabási, Scale-Free Networks: A Decade and Beyond, Science Magazine, 325, 412-413 (2009)

18. R. Albert. H. Jeong, A.L. Barabási, Diameter of the world-wide web, Nature, 401, 130131 (1999)

19. L. Muchnik, S. Pei, L.C. Parra, H. Makse, Origins of power-law degree distribution in the heterogeneity of human activity in social networks, Scientific Reports 3, 1783 (2013)

20. S. Virinchi, M. Pabitra, Link Prediction in Social Networks: Role of Power Law Distribution, Springer International Publishing (2016)

21. A.L. Barabasi, E. Bonabeau, Scale-Free Netwroks, Scientific American, 288, 50-59 (2003)

22. A. Claus, S. Kratzig, Optimal planning of network structures within an exchange area, European Journal of Operational Research, 7(1), 67-76 (1981) 